Ann. Zootech., I978, 27 (2), I8I-I94.

\title{
Echanges thermiques chez le porcelet nouveau-né : application de la méthode du bilan d'énergie
}

\author{
*P. BERBIGIER, **J. LE DIVIDICH et ***A. KOBILINSKY \\ * Station de Bioclimatologie, \\ ***Station de Biométrie, \\ Centre national de Recherches agronomiques, I.N.R.A., \\ 78000 Versailles (France) \\ ** Station de Recherches sur l'Elevage des Porcs, \\ Centre national de Recherches zootechniques, I.N.R.A., \\ 78350 Jouy-en-Josas (France)
}

\section{Résumé}

On a étudié, à température ambiante ( $\left.\mathrm{I} 7-\mathrm{I} 8^{\circ}{ }^{\circ} \mathrm{C}\right)$ et degré hygrométrique $(5 \mathrm{O}-60 \mathrm{p}$. cent) constant, l'influence des caractéristiques des porcelets nouveau-nés (poids, sexe, séchage de la peau à la naissance, températures rectale et cutanée initiales), sur l'évolution des températures rectale et cutanée (dos) pendant la première heure de vie. L'expérience a porté sur 72 porcelets, dont la moitié environ était séchée dès la naissance. Les mesures sont faites de ıo en Io mn depuis la naissance jusqu'à $\mathrm{I} h$. La température cutanée décroît de 7 à $9{ }^{\circ} \mathrm{C}$, et se stabilise en Io mr. La température rectale décroît de 2 à $3^{\circ} \mathrm{C}$ et se stabilise à $3^{\circ} \mathrm{mn}$.

Un modèle statistique permet :

- de montrer que les températures corporelles augmentent, à un moment donné après la naissance, avec le séchage, le poids et la température rectale initiale. Seuls ces facteurs jouent u1l rôle significatif. Le degré de signification est en général élevé $(\mathrm{P}<\mathrm{o}, \mathrm{I} \mathrm{p}$. cent);

- de prévoir l'évolution des températures corporelles d'un porcelet dont on connait ces caractéristiques.

On a également estimé la production de chaleur et la conductance des tissus, qui, globalement, augmentent avec le poids et le séchage.

\section{1. - Introduction}

Lorsque les conditions climatiques ambiantes ne varient pas, les cinétiques de décroissance des températures rectale et superficielle d'un porcelet pendant l'heure qui suit la mise bas dépendent de sa production de chaleur, des coefficients d'échange relatifs aux différentes voies de la thermolyse cutanée et pulmonaire, et du coefficient de conduction corporelle (Mount, I968). Quand un équilibre thermique est atteint (températures corporelles indépendantes du temps) on peut 
calculer les valeurs des paramètres traduisant les échangas cutanés en appliquant la théorie des échanges en régime stationnaire (MONTEITH, I973). En revanche, à l'instant de la naissance, on constate une décroissance des températures corporelles (NEWLAND, McMILLEN, REINEKE, I952); il est alors nécessaire d'introduire dans le calcul la variable temps (BERBIGIER et I)USSUEI, I977).

Parmi les phénomènes pouvant in fluencer la régulation thermique du nouveauné, certains, comme la circulation sanguine, l'utilisation des glucides et des acides gras libres du sang, les décharges hormonales, sont difficilement accessibles à la mesure : ce sont en général des phénomènes physiologiques. Cependant, certains peuvent être reliés à des mesures simples : on sait par exemple (PADALrkova, Holub, JEzkOVA, I972) que les réserves de glycogène du fœetus sont d'autant plus grandes que le stade de gestation est plus avancé : on peut donc penser que les porcelets nés avant terme ont des potentialités de thermorégulation plus faibles. De même, il est possible que le sexe ait une influence sur ces potentialités.

Contrairement aux phénomènes physiologiques, l'in fluence sur la thermorégulation des facteurs physiques, tels que l'inertie thermique du corps (poids à la naissance), la présence de liquides foetaux à la surface de la peau (évaporation), la température initiale du nouveau-né (égale à la température de l'utérus de la mère), est aisément mesurable.

Le facteur physique essentiel reste cependant le microclimat ambiant (DE IA Por'te Des VAux et Aumaitre, I967). Son effet, et notamment celui de la température de l'air, sur les températures corporelles et le confort thermique du nouveau-né, a été étudié précédemment (Dussuel et BERbigier, I978). Nous ne nous préoccuperons donc ici que de l'effet des caractéristiques propres aux animaux (surtout le poids et l'humidité de la peau), à conditions ambiantes constantes (température ambiante de l'ordre de I ${ }^{\circ} \mathrm{C}$, humidité d'environ $55 \mathrm{p}$. cent). L'analyse statistique permet de dégager des modèles prévisionnels de températures corporelles à différents temps après la mise bas, en fonction de ces caractéristiques.

\section{2. - Matériel et méthodes}

Les expériences ont été réalisées en porcherie de maternité de type "Big Dutchman ", thermostatée à I $8{ }^{\circ} \mathrm{C}$. Durant les expériences, la température et l'humidité de l'air au niveau des porcelets ont été mesurées à intervalles réguliers (toutes les $1 / 2$ heures).

On a étudié les cinétiques de températures corporelles de 72 porcelets de race Large White nés de II truies, pendant l'heure suivant l'expulsion. Pour ce faire, on mesure, 30 secondes après la mise bas, puis de Io en Io mn jusqu'à une heure, la température cutanée du dos du nouveau né (zone interscapulaire) par radiothermométrie; en même temps on détermine sa température rectale (sonde à thermistance enfoncée de $5 \mathrm{~cm}$ dans le rectum). Après la première mesure de température, les animaux sont pesés; un animal sur deux est séché le plus complètement possible avec du coton. Tous les animaux sont alors placés individuellement dans des boîtes en carton sèches isolées du sol, a fin de limiter les pertes par conduction. Les porcelets restent à jeun pendant la durée des mesures.

La température et 1'humidité de l'air sont déterminées au psychromètre au voisinage de l'animal. On a vérifié, au radiothermomètre, que la température moyenne des parois (boîtes et plafond rayonnant vers le porcelet, était toujours égale (à $\pm \mathrm{x}^{\circ} \mathrm{C}$ près) à la température de l'air. 


\section{3. - Théorie}

\section{I. - Traitement statistique}

Il permet de préciser l'effet des facteurs suivants : poids vif, sexe, séchage à la mise-bas, température rectale initiale du nouveau-né, sur l'évolution des températures corporelles. Nous avons choisi d'étudier les températures rectales et de surface à Io mn (stabilisation de la température de surface) et à $60 \mathrm{mn}$ (équilibre thermique réalisé).

On introduit également un facteur " effet de la mère ". L'effet de la maturité physiologique des porcelets, qui ne peut être dissocié de l'effet de la mère et ne peut être testé que sur quelques portées (peu de mise-bas ont lieu à plus d'un jour d'écart de la date prévue) n'a pas été introduit dans le modèle.

\section{II. Modèle}

-- Pour désigner les variables et facteurs, nous utiliserons les symboles suivants: $y$ : variable étudiée (température rectale ou de surface à ro mn et $60 \mathrm{mn}$ ).

Variables expliquées:

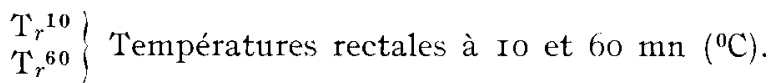

$\left.\begin{array}{c}\mathrm{T}_{8}{ }^{10} \\ \mathrm{~T}_{s}{ }^{60}\end{array}\right\}$ Températures de surface à ro et $60 \mathrm{mn}\left({ }^{\circ} \mathrm{C}\right)$.

Facteurs quantitalifs (Covariables):

$\mathbf{P}$ : poids vif du porcelet $(\mathrm{kg})$.

$\mathrm{T}_{s}{ }^{0}$ : température de surface initiale (30 s après $1^{\prime}$ expulsion) $\left({ }^{0} \mathrm{C}\right)$.

$\mathrm{I}_{r}{ }^{0}$ : température rectale initiale (30 s après l'expulsion) $\left({ }^{\circ} \mathrm{C}\right)$.

Facteurs qualitatifs:

$i$ : numéro de la truie $(i: \mathbf{I}, 2, \ldots, \mathrm{I} I$ ) (Effet de la mère).

$j: \operatorname{sexe}(j=\mathrm{I}:$ mâles, $j=0$ : femelles).

$k$ : traitement ( $k=\mathrm{I}$ : animaux séchés, $k=0$ : non séchés).

- Le modèle utilisé est le modèle statistique linéaire classique. On y introduit des termes pour l'interaction entre le traitement et les autres facteurs : ainsi les covariables $\mathrm{P}, \mathrm{T}_{s}{ }^{0}, \mathrm{~T}_{r}{ }^{0}$ interviennent de façon linéaire, mais avec un coefficient pouvant dépendre du traitement $k$.

Le modèle posé est donc le suivant :

(I) $\quad \mathrm{E}\left(y / i, j, k, \mathrm{P}, \mathrm{T}_{r}^{0}, \mathrm{~T}_{s}^{0}\right)=\mu+\alpha_{i}+\beta_{j}+\gamma_{k}+\lambda \mathrm{P}+\phi \mathrm{T}_{r}{ }^{0}+\psi_{s} \mathrm{~T}^{0}+\delta_{j k}+\lambda_{k} \mathrm{P}$ $+\phi_{k} \mathrm{~T}^{0}{ }^{0}+\psi_{k} \mathrm{~T}_{s}{ }^{0}$

où $\mathrm{E}\left(y / i, j, k, \mathrm{P}, \mathrm{T}_{r}{ }^{0}, \mathrm{~T}_{s}{ }^{0}\right)$ est l'espérance de $y$ pour un porcelet de mère $i$, de sexe $i$, ayant reçu le traitement $k$, de poids vif $\mathrm{P}$, de températures initiales $\mathrm{T}_{r}{ }^{0}$ et $\mathrm{T}_{s}{ }^{0}$. $\mu, \alpha_{i}, \beta_{j}, \gamma_{k}, \lambda, \phi, \psi$, etc..., sont les paramètres inconnus du modèle. La présence des termes $\lambda_{k}, \phi_{k}$, पेk traduit une possible hétérogénéité des régressions sur $\mathrm{P}, \mathrm{T}_{r}{ }^{0}$, $\mathrm{T}_{s}{ }^{0}$, entre les traitements. 
- L'application de ce modèle aux observations du plan d'expérience (afin d'estimer les paramètres) s'écrit sous la forme plus classique :

$$
\begin{aligned}
y_{i j k, l}= & \mu+\alpha_{i}+\beta_{j}+\gamma k+\lambda\left(\mathrm{P}_{i j k l}-\overline{\mathrm{P}}\right)+\phi\left(\mathrm{T}_{r}{ }_{i j k l}-\overline{\mathrm{T}_{r}{ }^{0}}\right)+\psi\left(\overline{\mathrm{T}_{s}{ }_{i j k l}}-\overline{\mathrm{T}_{s}{ }^{0}}\right)+ \\
& +\delta_{j k}+\lambda_{k}\left(\mathrm{P}_{i j k l}-\mathrm{P}\right)+\phi_{k}\left(\mathrm{~T}_{r}{ }^{0_{i j k l}}-\overline{\left.\mathrm{T}_{r}{ }^{0}\right)}+\psi_{k}\left(\mathrm{~T}_{s}{ }^{0}{ }_{i j k l}-\overline{\left.\mathrm{T}_{s}{ }^{0}\right)}+\varepsilon_{i j k l}\right.\right.
\end{aligned}
$$

où $l$ est l'indice permettant de repérer le porcelet.

\subsection{Tests. Réduction du modèle}

Pour tester l'effet d'un facteur, on se ramène généralement à un niveau constant des autres facteurs en ajustant le test correspondant. Pratiquement, un tel test s'obtient à partir de l'augmentation de variance résiduelle provoquée par la suppression du facteur étudié dans un modèle où figurent les autres facteurs.

Cependant une procédure n'utilisant que de tels tests ajustés peut amener une perte d'information importante. Prenons l'exemple des facteurs $\mathrm{T}_{r}{ }^{0}$ et $\mathrm{T}_{s}{ }^{0}$, ici extrêmement liés. Analyser l'effet d'un de ces deux facteurs, l'autre étant fixé, revient à analyser l'effet du gradient de température initiale. Pour prouver la rémanence de 1'effet température initiale Io $\mathrm{mn}$ ou $60 \mathrm{mn}$ après la mise-bas, il faut tester la régression de $\mathrm{T}_{r}{ }^{0}$ sans $1^{\prime}$ ajuster pour $\mathrm{T}_{s}{ }^{0}$ (ou inversement ${ }^{\prime} \mathrm{T}_{s}{ }^{0}$ non ajusté pour $\mathrm{T}_{r}{ }^{0}$ ).

La méthode utilisée ici élimine progressivement du modèle (I) les termes dont la suppression n'augmente pas significativement la variance résiduelle. Un facteur figurant dans le modèle " minimal " ainsi obtenu a donc un effet significatif, qui ne peut se réduire à l'effet direct de l'un des autres facteurs de ce même modèle. L'information apportée par cette seule expérience ne permet cependant pas de dissocier l'effet de deux facteurs très liés, dont l'un ne figure pas dans le modèle minimal (cas de $\mathrm{T}_{r}{ }^{0}$ et $\mathrm{T}_{s}{ }^{0}$, de l'effet truie et des facteurs liés aux conditions de mise-bas).

\section{I3. Modèle prédictif. Estimation}

En utilisant cette même méthode d'élimination, mais à partir du modèle

$$
\begin{aligned}
& \mathrm{E}\left(y / j, k, \mathrm{P}, \mathrm{T}_{s}{ }^{0}, \mathrm{~T}_{r}{ }^{0}\right)=\mu+\beta_{j}+\gamma_{k}+\lambda \mathrm{P}+\phi \mathrm{T}_{r}{ }^{0}+\mathrm{T}_{s}{ }^{0}+\delta_{j k}-\lambda_{k} \mathrm{P}+ \\
& \phi_{k} \mathrm{~T}_{s}{ }^{0}+\psi_{k} \mathrm{~T}_{r}{ }^{0}
\end{aligned}
$$

qui ne comporte pas d'effet truie, on obtient un modèle permettant de prédire l'évolution de la température chez un porcelet nouveau-né.

L'estimation des paramètres de ce modèle, plus précise que celle des paramètres du modèle complet (2), permet une prédiction meilleure lorsque l'on s'écarte des conditions strictes de l'expérience.

\section{2. - Traitement physique du quasi-équilibre thermique obtenu à partir de $40 \mathrm{mn}$ après la mise-bas}

$40 \mathrm{mn}$ après la mise-bas (fig. I), le régime d'échanges est pratiquement stationnaire. 'Tous les animaux sont alors secs. La thermolyse et la conduction du corps peuvent être données par les équations suivantes (BERBIGIER, I975).

$$
\phi_{\mathrm{M}}=\left(\mathrm{C}_{r}+\mathrm{C}_{c}\right)\left(\mathrm{T}_{s}-\mathrm{T}_{a}\right)
$$

$$
\mathrm{C}_{i}=\left(\mathrm{C}_{r}+\mathrm{C}_{c}\right)\left(\mathrm{T}_{s}-\mathrm{T}_{a}\right) /\left(\mathrm{T}_{r}-\mathrm{T}_{s}\right)
$$


$\left(\mathrm{C}_{r}+\mathrm{C}_{c}\right)$ : coefficient d'échange radiatif $\left(\mathrm{C}_{r}\right)-$ convectif $\left(\mathrm{C}_{c}\right)\left(\mathrm{Wm}^{-2}{ }^{0} \mathrm{C}^{-1}\right)$.

$\mathrm{T}_{s}$ : Température de surface $\left({ }^{\circ} \mathrm{C}\right)$.

$\mathrm{T}_{r}$ : Température rectale $\left({ }^{0} \mathrm{C}\right)$.

$\mathrm{T}_{a}$ : Température ambiante $\left({ }^{\circ} \mathrm{C}\right)$.

$\phi_{\mathrm{M}}$ : Densité de flux de thermolyse $\left(\mathrm{Wm}^{-2}\right)$.

$\mathrm{C}_{i}$ : Coefficient de conduction du corps $\left(\mathrm{Wm}^{-2}{ }^{\circ} \mathrm{C}^{-1}\right)$.

Toutefois dans l'estimation de $\phi_{M}$, nous ne tenons pas compte des pertes latentes qui, pour une température de l'air de $I 7{ }^{\circ} \mathrm{C}$, représentent moins de Io $\mathrm{p}$. cent des pertes totales chez le porcelet nouveau-né (Mount, I962). Les pertes par conduction sont également négligées. Elles représentent dans ces conditions expérimentales, moins de 15 p. cent des pertes totales (MounT, I967). En outre, nos porcelets ont toujours adopté une posture ramassée, ce qui réduit d'autant les pertes par cette voie.

\section{4. - Résultats}

4.I. - Influence des jacteurs liés à l'animal sur les cinétiques de températures

\section{II. Description des données}

Le tableau I permet de vérifier que les conditions ambiantes sont peu variables et ne jouent aucun rôle dans les écarts observés.

\section{TABLEAU I}

Temperature ( $T a$ ) et pression partielle de vapeur d'eau ( $P a$ ) ambiantes moyennes, velatives aux diférents traitements et classes de poids

Mean ambient temperature $(T)$ and partial pressure of water vapour $(P a)$ for diflerent treatments and weight classes

\begin{tabular}{|c|c|c|c|c|c|c|c|c|}
\hline $\begin{array}{l}\text { Traitement } \\
\text { Treatmont }\end{array}$ & \multicolumn{4}{|c|}{$\begin{array}{l}\text { Animal séché } \\
\text { Dried animal }\end{array}$} & \multicolumn{4}{|c|}{$\begin{array}{l}\text { Animal non séché } \\
\text { Undried animal }\end{array}$} \\
\hline $\begin{array}{l}\text { Classe } \\
\text { de poids } \\
\text { Weight } \\
\text { class }\end{array}$ & $\begin{array}{l}\text { Nombre } \\
\text { d'ani- } \\
\text { maux } \\
\text { Numbcr } \\
\text { of } \\
\text { animals }\end{array}$ & $\begin{array}{c}\text { Poids } \\
\text { moyen de } \\
\text { la classe } \\
\text { (kg) } \\
\text { Mean } \\
\text { weight } \\
\text { of class } \\
(\mathrm{kg})\end{array}$ & $\begin{array}{c}T a \\
\left({ }^{\circ} \mathrm{C}\right)\end{array}$ & $\begin{array}{c}\mathrm{Pa} \\
\text { (pascal) } \\
P a \\
\text { (pascal) }\end{array}$ & $\begin{array}{l}\text { Nombre } \\
\text { d'ani- } \\
\text { Inaux } \\
\text { Number } \\
\text { of } \\
\text { animals }\end{array}$ & $\begin{array}{c}\text { Poids } \\
\text { moyen de } \\
\text { la classe } \\
(\mathrm{kg}) \\
\text { Mean } \\
\text { Weight } \\
\text { of class } \\
(\mathrm{kg})\end{array}$ & $\begin{array}{c}\mathrm{Ta} \\
\left({ }^{\circ} \mathrm{C}\right) \\
T a \\
\left({ }^{\circ} \mathrm{C}\right)\end{array}$ & $\begin{array}{c}\mathrm{Pa} \\
\text { (pascal) } \\
P a \\
(\text { pascal) }\end{array}$ \\
\hline$P \leqslant I \mathrm{~kg} \cdot \cdot \cdot \cdot$. & 8 & 0,862 & I $7,3^{2}$ & $93^{6}$ & 8 & 0,852 & 17,72 & I 177 \\
\hline $\mathrm{I} \mathbf{k g}<\mathbf{P} \leqslant \mathrm{I}, \geq \mathrm{kg}$. & I 3 & I , I 4 I & I 6,90 & I $13^{6}$ & IO & I , I I 8 & I 7,87 & I 077 \\
\hline $\mathrm{I}, 2 \mathrm{~kg}<\mathrm{P}<\mathrm{I}, 4 \mathrm{~kg}$ & 6 & $\mathrm{I}, 3 \mathrm{I} 2$ & I 6,93 & I 124 & ro & $\mathrm{I}, 3^{\mathrm{O} 2}$ & 17,98 & I. 008 \\
\hline $\mathrm{I}, 4 \mathrm{~kg}<\mathrm{P}$ & 8 & $\mathrm{I}, 564$ & 17,70 & I 109 & 9 & $\mathrm{I}, 488$ & I 7,47 & I I 39 \\
\hline $\begin{array}{l}\text { Total ou moyenne. } \\
\text { Total or mean. . . }\end{array}$ & 35 & $\mathbf{I}, 203$ & 17,18 & 1082 & 37 & $\mathrm{I}, 203$ & I 7,77 & I 096 \\
\hline
\end{tabular}


L'allure des cinétiques de température est représentée sur les figures I $a$ et $\mathbf{I} b$, pour les animaux séchés ou non séchés, et par classe de poids. La température de surface décroît brutalement avant Io $\mathrm{mn}$ puis devient stationnaire ou augmente légèrement jusqu'à $60 \mathrm{mn}$. La température rectale décroît plus lentement et plus régulièrement, se stabilise au bout de 20 ou $30 \mathrm{mn}$, puis reste également stationnaire ou en légère augmentation.

Le séchage réduit la chute de température. Cependant, du fait de l'inertie thermique corporelle l'effet s'observe moins vite sur la température rectale que
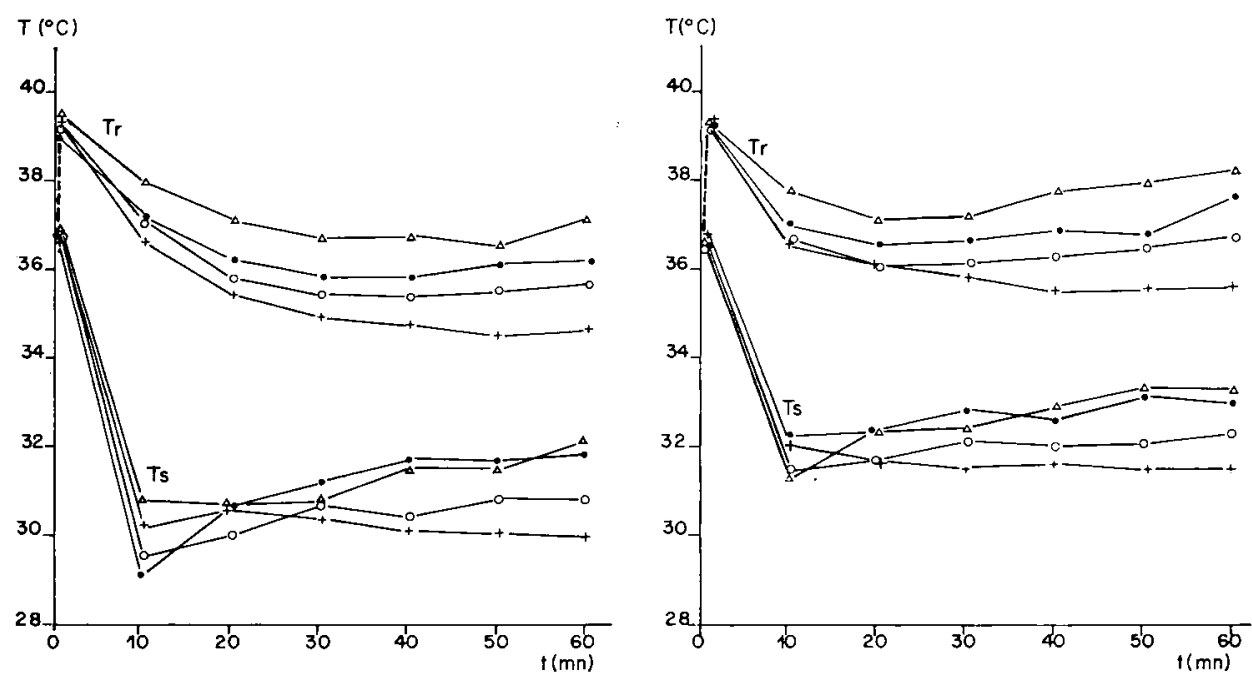

Fig. 1. - Cinétiques des températures rectales $\left(T_{r}\right)$ et de surface $\left(T_{s}\right)$ par classe de poids, des porcelets nouveau-nés

Kinetics of rectal $\left(T_{r}\right)$ and surface $\left(T_{s}\right)$ temperatures of new-born piglets for different weight $(P)$ classes

$\begin{array}{llll}+ & \mathrm{P} & < & \\ \mathrm{O} & \mathrm{I} 000 & \mathrm{I} 000 \mathrm{~g} \\ \mathrm{I} 200 \mathrm{~g} \\ \mathrm{P} & \mathrm{I} 200<\mathrm{I} 400 \mathrm{~g} \\ \mathrm{P} & & \mathrm{P} \geqslant \mathrm{I} 400 \mathrm{~g}\end{array}$

a) Animaux non séchés à la mise-bas (Animals kept wet at farrowing).

b) Animaux séchés à la mise-bas (Animals wiped dry at jarrowing).

sur la température de surface. Une heure après la naissance, le séchage initial des porcelets se traduit par un gain de $I^{\circ} \mathrm{C}$ sur $T_{s}$ et $T_{r}$.

Les deux températures $T_{s}$ et $T_{r}$ sont d'autant plus élevées que l'animal est lourd. Cependant, pour la température de surface, cette tendance ne se confirme qu'au bout de $30 \mathrm{mn}$. Entre 850 et I $500 \mathrm{~g}$, une augmentation de roo g de poids corporel provoque une augmentation d'environ $0,4{ }^{\circ} \mathrm{C}$ de la température rectale prise à $60 \mathrm{mn}$. Par contre, il semble qu'au-dessus de I $200 \mathrm{~g}$, la température de surface ne dépende plus du poids, que l'animal ait été séché ou non. On constate enfin, qu'il n'y a pas d'interaction traitement-poids. Les différences entre les températures relatives aux 4 classes de poids sont les mêmes quel que soit le traitement. 


\section{I2. - Analyse statistique}

Elle a été effectuée selon la méthode décrite plus haut. Les résultats sont donnés dans le tableau 2.

I,es termes correspondant aux interactions, à l'effet du sexe, à la température de surface initiale ont été éliminés dans tous les cas. Les autres termes éliminés sont ceux pour lesquels le test (respectivement 1'estimation) est absente. Ainsi le modèle utilisé pour prédire $\mathrm{T}_{r}{ }^{10}$ est :

$$
\begin{array}{r}
\mathrm{T}_{r}{ }^{10}=\widehat{\mu}+\widehat{\lambda}(\mathrm{P}-\overline{\mathrm{P}})+\widehat{\phi}\left(\mathrm{T}^{0}-\mathrm{T}_{r^{0}}\right)+\varepsilon \\
\text { où } \hat{\mu}=37,09, \hat{\lambda}=\mathrm{I}, 807, \widehat{\phi}=0,889, \operatorname{Var}(\varepsilon)=0,32 .
\end{array}
$$

Le centrage de $\overline{\mathrm{P}}$ et $\overline{\mathrm{T}_{r}}{ }^{0}$ permet de donner un sens à la constante $\hat{\mu}$ qui devient ainsi la valeur de la prédiction pour un procelet de poids moyen, ayant une température initiale rectale moyenne. La contrainte adoptée pour $\widehat{\mu}(\hat{\mu}=0)$ lorsque le traitement est présent a également pour objet de donner un sens à $\widehat{\gamma}_{1}$ et $\widehat{\gamma}_{2}$. $\widehat{\gamma}_{1}$ est donc la valeur prédite pour un porcelet de poids moyen, de température rectale initiale moyenne, qui est séché à la mise-bas.

L'étude détaillée des résultats sera reprise dans la discussion. Cependant, on voit globalement que les facteurs agissant de façon prépondérante sur la température corporelle du porcelet sont le poids à la naissance, l'état de mouillage de la peau, la température rectale initiale. L'influence de la mère est faible. L'influence du sexe n'est jamais significative. Il n'y a pas d'interaction significative entre le traitement (séchage) et les autres facteurs. I a température de surface (surtout à Io $\mathrm{mn}$ ) est moins liée à l'ensemble des facteurs que la température rectale.

\section{2. - Étude physique des pertes de chaleur après dessèchement initial}

La thermolyse $\left(\phi_{\mathrm{M}}\right)$ et le coefficient de conduction corporelle $\left(\mathrm{C}_{i}\right)$ ont été estimés à partir du temps $t=40 \mathrm{mn}$ correspondant à l'établissement de l'équilibre thermique (équations 3 et 4 ). Le coefficient d'échange convectif et radiatif $\left(\mathrm{C}_{c}+\mathrm{C}_{r}\right)$ utilisé pour le calcul, a été déterminé à postériori dans les conditions de l'expérience selon une méthodologie décrite précédemment (BERBIGIER, I975; BERBIGIER et Dussuei, I 977) à l'aide de porcelets mort-nés, mouillés et réchauffés à $39^{\circ} \mathrm{C}$. Ce coefficient variant peu avec le poids des animaux (MON'TEITH, I973), la valeur trouvée, soit : $\mathrm{C}_{c}+\mathrm{C}_{r}=6,77 \mathrm{Wm}^{-2}{ }^{0} \mathrm{C}^{-1}$, est donc appliquée à tous les porcelets.

\subsection{I. Étude de $\phi_{\mathrm{II}}$}

La production de chaleur (équation 3) est directement proportionnelle à l'écart température de surface - température de 1'air. Toutefois, en raison de la localisation de la mesure de la température cutanée, l'équation 3 ne permet d'estimer que la thermolyse dorsale.

$T_{a}$ variant peu (cf. tab1. I), l'influence des différents facteurs étudiés sur $\phi_{\text {II }}$ sera très semblable à celle observée sur $\mathrm{T}_{s}{ }^{60}$ où les seuls effets significatifs sont le poids et le séchage. La gamme des poids étudiés est faible, et une régression linéaire 


\section{TABIEAU 2}

Réduction des modèles statistiques permettant l'estimation des températures cutanées $\left(T_{s}\right)$ et rectales $\left(T_{r}\right)$, Io $m n$ et $60 \mathrm{mn}$ après la mise-bas

Reduction of statistical models for estimation of shin surface $\left(T_{s}\right)$ and rectal $\left(T_{r}\right)$ temperatures Io $m n$ and $60 \mathrm{mn}$ after farrowing

Variance totale.

(Total variance)

Variance résiduelle du modèle I (avec effet truie) . . . . . . . . . . . . . . . .

(Residual variance of model $I$ (with sow eflect))

Variance résiduelle du modèle minimal déduit

du modèle I. . . . . . . . . . . . .

(Residual variance of minimum model obtained from model I)

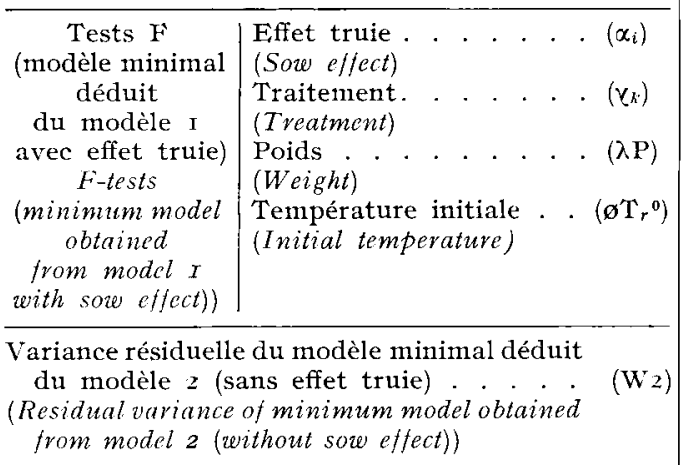

\begin{tabular}{|c|c|c|c|c|c|}
\hline & $\underset{(\text { Constant })}{\text { Constante }} \cdot \cdot \cdot \cdot \cdot \cdot \cdot(\widehat{u})$ & o & 37,09 & 0 & 0 \\
\hline $\begin{array}{l}\text { Estimation } \\
\text { des paramètres } \\
\text { (modèle mininal }\end{array}$ & $\mid \begin{array}{l}" \operatorname{Sec} \| * \cdot\left(\widehat{\Upsilon}_{1}\right) \\
(" D r y \|)\end{array}$ & $3^{1}, 74$ & - & 32,46 & 37,03 \\
\hline $\begin{array}{c}\text { déduit } \\
\text { du modèle } 2 \\
\text { sans effet truie) }\end{array}$ & \begin{tabular}{l|l} 
(Treatment) & $\begin{array}{l}\text { "Humide ". } \\
\text { ("Wet" }\left(\widehat{\aleph_{2}}\right)\end{array}$
\end{tabular} & $3^{\circ}, \mathrm{OI}$ & 一 & $3 r, 27$ & 35,92 \\
\hline $\begin{array}{c}\text { Estimation } \\
\text { of parameters } \\
\text { (minimum model }\end{array}$ & $\begin{array}{l}\text { Coefficient du Poids (P). }(\widehat{\lambda}) \\
\text { (Coefficient of Weight }(P)\end{array}$ & - & 1,807 & 2,997 & $3,83^{\circ}$ \\
\hline $\begin{array}{c}\text { obtained } \\
\text { from model } 2 \\
\text { without sow effect) }\end{array}$ & $\begin{array}{l}\text { Coefficient de la tempéra- } \\
\text { ture initiale }\left(\mathrm{T}_{r}^{0}\right) \cdot{ }^{\circ} \cdot(\widehat{\varnothing}) \\
\text { Coefficient of initial tempe- } \\
\text { ratuve }\left(T_{r}^{0}\right)\end{array}$ & 0,67 & 0,889 & -- & 0,755 \\
\hline
\end{tabular}

Note : - Les nombres entre crochets indiquent le ou les degrés de libertés associés à la variance ou au $F$. — * : significatif à $5 \mathrm{p}$. cent; ** : significatif c̀ $\mathrm{x} \mathrm{p}$. cent; ***: significatif à $\mathrm{x}$ p. mille.

— $\lambda$ et $\emptyset$ doivent être appliquées à $\mathrm{P}-\overline{\mathrm{P}}$ et $\mathrm{T}_{r}{ }^{0}-\overline{\mathrm{T}}_{r}^{0}$ où $\overline{\mathrm{P}}=\mathrm{I} .202 ;{\overline{\mathrm{T}_{r}}}^{0}=39.33$.

Note: - Numbers between brackets give the degrees of freedom associated to variance or $F$-value.

— 5 p. cent significance; ** I p. cent significance; *** I p. cent significance.

- $\lambda$ and $ø$ must be applied to $P-\bar{P}$ and $T_{r}{ }^{0}-\overline{T_{r}{ }^{0}}$ where $\bar{P}=1.202 ; \bar{T}_{r^{0}}{ }^{-}=39.33$. 
suffit à rendre compte de cet effet. Avec trois données par animal (40-50-60 mn), le calcul (équation 3) donne:

Animaux séchés (I05 données) :

$$
\phi_{\mathrm{M}}\left(\mathrm{W}^{\mathrm{T}} \cdot \mathrm{m}^{-2}\right)=\mathrm{I} 2,89 \mathrm{P}(\mathrm{kg})+90,53 \quad r=0,28(\mathrm{P}<0, \mathrm{OI})
$$

Animaux non séchés (I I données) :

$$
\phi_{\mathrm{Mr}}\left(\mathrm{Wm}^{-2}\right)=2 \mathrm{I}, \mathrm{I} 6 \mathrm{P}(\mathrm{kg})+67,42 \quad r=0,46(\mathrm{P}<0, \mathrm{OI})
$$

La comparaison des deux équations montre seulement une différence significative $(\mathrm{P}<0,00 \mathrm{I})$ entre les ordonnées à l'origine. Ceci confirme les résultats obtenus sur $\mathrm{T}_{\delta}{ }^{60}$ (tab1. 2), à savoir que les effets "traitement " (ordonnées à l'origine) et poids (coefficients de corrélation) sur $\phi_{M}$ sont très significatifs, mais que l'interaction poids-traitement ne l'est pas (comparaison des pentes).

Il faut cependant remarquer :

- que $\phi_{\mathrm{M}}$ semble moins lié que $\mathrm{T}_{s}{ }^{60}$ au facteur poids. On peut expliquer ceci par la variabilité supplémentaire due d'une part à l'introduction de $\mathrm{T}_{\boldsymbol{a}}$ dans le calcul, d'autre part au fait que 1'on utilise trois données par animal (effet du temps);

- que, bien que la différence ne soit pas significative, l'effet du poids semble plus important sur les animaux non séchés. Il semble que, lorsque le stress thermique augmente (évaporation) les petits animaux soient plus désavantagés que les gros et que leur production de chaleur diminue plus.

\subsection{2. Étude de $C_{i}$ (calculé à partir de l'équation 4)}

Les pentes des droites de régression $\mathrm{C}_{i}=\mathrm{F}$ (Poids) ne sont, ni pour les animaux secs ni pour les animaux mouillés, significativement différentes de o. On fait donc une moyenne des valeurs obtenues pour chaque traitement (tabl. 3).

Le séchage à la naissance a donc une influence bénéfique très nette sur la conduction du corps, c'est-à-dire l'intensité de la circulation sanguine périphérique.

TABI,EAU 3

Coefficient de conduction thermique du corps en fonction du traitement

\begin{tabular}{|c|c|c|}
\hline Traitement - Treatmont & Sec $-I r^{\prime} y$ & Mouillé - Wet \\
\hline Nonnbre de données (Number of data). . . . & IO5 & I I I \\
\hline $\mathrm{Ci}\left(\mathrm{W} . \mathrm{m}^{-2}{ }^{\circ} \mathrm{C}^{-1}\right)$. & 25,03 & 20,65 \\
\hline$\Delta \mathrm{Ci}$ (écart type : W. $\left.\mathrm{m}^{-2}{ }^{\circ} \mathrm{C}^{-1}\right)($ Standarderror) & $5,6 \mathrm{I}$ & 5, I 9 \\
\hline $\begin{array}{l}\text { Degré de signification de la différence de } \\
\text { moyennes (Significance of difference between } \\
\text { means)... . . . . . . . . . . . . . . . . . . . . }\end{array}$ & \multicolumn{2}{|c|}{$t=5,95 \quad(\mathrm{P}<0,00 \mathrm{I})$} \\
\hline
\end{tabular}
Body thermal conductance related to treatment 


\section{5. - Discussion}

\section{I. - Allure des courbes}

On voit (fig. I) que la chute initiale de la température de surface est beaucoup plus rapide et beaucoup plus intense que celle de la température rectale. Cependant l'équilibre se fait plus vite (moins de Io min) à la surface qu'au niveau du rectum (30 $\mathrm{mn})$. Cet équilibre est-il purement physique (réduction des échanges thermiques due à la disparition des liquides fotaux, et changement de régime de convection) ou physiologique (thermorégulation au niveau de la peau)? Les deux explications se complètent. En effet, on a montré (DUSSUEL et BERBIGIER, I978) que la thermorégulation du nouveau né se faisait essentiellement au niveau de la peau; d'autre part, on sait (BERBIGIER et DUSSUEL, I977) que le séchage et le passage de la convection libre à la convection forcée (réduction des pertes) intervenaient aux alentours de $\mathrm{s}$ mn.

Il semble donc qu'il existe une thermorégulation au niveau de la peau, qui est facilitée par des phénomènes physiques intervenant environ $10 \mathrm{mn}$ après la naissance.

Par contre, 1'allure de la courbe d'évolution de la température rectale, de type exponentiel décroissant, montre qu'il n'y a probablement pas de thermorégulation à ce niveau. Cette courbe est typique des phénomènes de diffusion de la chaleur dans un solide inerte.

\section{2. - Influence des différents facteurs}

L'analyse statistique montre que seuls le traitement (séchage à la naissance), le poids des animaux (Stanton, Brown, MUELIER, I973) et la température rectale initiale (supposée égale à la température de l'utérus de la mère) interviennent de façon significative. L'influence de la mère, en dehors de la température de l'utérus, ne se manifeste que sur la température de surface à Io mn, et de façon peu significative (à 5 p. cent). On n'a pas trouvé d'interactions entre le traitement et les autres facteurs.

\subsection{Influence du poids à la naissance}

Elle se manifeste déjà au bout de Io mn pour la température rectale (significative à $o, I$ p. cent). Par contre, elle n'intervient que plus tard (fig. I) sur la température de surface. A $60 \mathrm{mn}$, son effet est significatif à 0, I p. cent sur les deux températures. La figure I montre qu'à $60 \mathrm{mn}$, une augmentation de poids de Ioo $\mathrm{g}$ s'accompagne d'une augmentation de $\mathrm{T}_{s}{ }^{60}$ de $0,3{ }^{\circ} \mathrm{C}$ et une augmentation de $\mathrm{T}_{r}{ }^{60}$ de $0,38^{\circ} \mathrm{C}$. Cependant, le modèle linéaire n'est probablement pas exact pour $\mathrm{T}_{s}{ }^{60}$ : en effet, au dessus de $\mathrm{I}, 3 \mathrm{~kg}$, le poids n'a plus d'influence. On atteint un seuil de température de surface; ceci peut être mis en parallèle avec l'effet du poids sur $\mathrm{T}_{s}$ trouvé par DUSSUEL et BERBIGIER (I978) à température ambiante plus élevée (supérieure à $23^{\circ} \mathrm{C}$ ) : le seuil est alors obtenu pour un poids de $0,85^{\circ} \mathrm{kg}$.

\subsection{Influence du séchage à la naissance}

Elle apparaît dès Io mn sur la température de surface (significative à o, I $\mathrm{p}$. cent, ce qui est normal, car le séchage modifie d'abord les échanges superficiels : 
diminution de l'évaporation) : du fait de l'inertie thermique du corps, son' effet n'apparaît qu'ensuite sur la température rectale. A $60 \mathrm{mn}$, le séchage initial a un effet rémanent très significatif $(0, \mathrm{r} p$. cent $)$ : à poids égal, les températures rectales et superficielles des animaux séchés sont supérieures d'environ ${ }^{\circ}{ }^{\circ} \mathrm{C}$ à celles des animaux non séchés.

\subsection{Influence de la température rectale initiale}

Elle n'est jamiais significative sur les températures de surface, qui dépendent beaucoup plus de l'équilibre des échanges avec le milieu ambiant que des conditions initiales de température. Par contre, lorsque deux porcelets ont entre eux une différence de température rectale de $\mathrm{I}{ }^{\circ} \mathrm{C}$ à la naissance $\left(\mathrm{T}_{r}{ }^{0}\right)$, cette différence subsiste jusqu'à $60 \mathrm{mn}$. (différence de $0,89^{\circ} \mathrm{C}$ à Io $\mathrm{mn}$, de $0,7^{6}{ }^{\circ} \mathrm{C}$ à $60 \mathrm{mn}$ : cf. tabl. 2).

\section{3. - Modèles prévisionnels}

Bien que ne tenant pas compte des conditions thermiques ambiantes, ils sont intéressants dans la mesure où la température de 1 'air $\left(\mathrm{I}_{7,5}{ }^{\circ} \mathrm{C}\right)$ correspond à peu près à la valeur à laquelle le métabolisme " de sommet " est obtenu pour des porcelets d'un jour (NichEI,MANN, BARNick, LYS, I974). Ainsi ces modèles décrivent les variations de température corporelle à la limite de la thermorégulation vers les basses températures, et permettent de prévoir les valeurs minimales atteintes.

Les modèles obtenus à $60 \mathrm{mn}$, lorsque l'équilibre thermique est établi, fournissent les valeurs suivantes :
$\mathrm{T}_{8}{ }^{60}\left({ }^{\circ} \mathrm{C}\right)=32,5+3,00(\overline{\mathrm{P}}-\mathrm{P})$ Animal séché.
$\mathrm{T}_{8}{ }^{60}\left({ }^{\circ} \mathrm{C}\right)=3 \mathrm{~T}, 3+3,00(\overrightarrow{\mathrm{P}}-\mathrm{P})$ Animal non séché.
$\mathrm{T}_{s}{ }^{60}\left({ }^{\circ} \mathrm{C}\right)=37,0+3,83(\overline{\mathrm{P}}-\mathrm{P})+0,76\left(\mathrm{~T}_{r}-\overline{\mathrm{T}^{\circ}}{ }_{r}\right)$ Animal séché.
$\mathrm{T}_{r}{ }^{60}\left({ }^{\circ} \mathrm{C}\right)=35,9+3,83(\overline{\mathrm{P}}-\mathrm{P})+0,76\left(\mathrm{~T}^{\circ}{ }_{r}-\overline{\mathrm{T}^{\circ}}{ }_{r}\right)$ Animal non séché.
$\overline{\mathrm{P}}=\mathrm{I}, 202 \mathrm{~kg}$ est $1 \mathrm{e}$ poids moyen des animaux étudiés.
$\overline{\mathrm{T}_{r}{ }^{0}}=39,33^{\circ} \mathrm{C}$ est leur température rectale initiale moyenne.

\section{4. - Pertes de chaleur et coefficients de conduction du corps}

Leur estimation repose sur la détermination, sur des porcelets morts placés dans les conditions de l'expérience (boites de carton, température ambiante de I $7^{\circ} \mathrm{C}$ ), du coefficient d'échange radiatif-convectif.

On obtient: $\mathrm{C}_{r}+\mathrm{C}_{c}=6,77 \mathrm{~W} \cdot \mathrm{m}^{-2}{ }^{0} \mathrm{C}^{-1}$.

Ce coefficient est un coefficient local (surface du dos). Les valeurs estimées sont donc des valeurs locales en ce point. Dans les conditions précises de l'expérience, ce coefficient ne semble pas varier avec le poids de l'animal.

La valeur trouvée est très faible : elle correspond pratiquement à la diffusion dans une couche d'air calme (MONTEITH, I973; BERBIGIER, I975) : celle-ci est peut-être emprisonnée dans le pelage, même clairsemé. JoyCE, BLAXTER et PARK (I966) trouvent des valeurs similaires pour les moutons. 
On n'a calculé que les pertes de chaleur sensible par convection et radiation. Les autres voies de la thermolyse (évaporation et conduction solide) représentent (Moun', I967; BERBIGIER, I978) de Io à $20 \mathrm{p}$. cent des pertes totales, donc de la production de chaleur. Ce calcul approché de la production de chaleur montre que pour un porcelet de I $300 \mathrm{~g}$ non séché, le métabolisme de sommet est de l'ordre de IIO Wm-2 (NICHELMANN, BARNICK, Lys, I974, porcelets d'un jour : environ Ioo $\mathrm{Wm}^{-2}$ ). Le séchage augmente la production de chaleur d'environ $7 \mathrm{Wm}^{-2}$. Celle-ci augmente significativement avec le poids des animaux.

I e coefficient de conduction du corps $\left(\mathrm{C}_{i}\right)$ augmente lorsqu'on sèche les animaux : le séchage a donc un effet favorable sur la circulation sanguine périphérique. Il faut signaler qu'à condition d'utiliser la valeur de $\mathrm{C}_{r}+\mathrm{C}_{c}$ que nous avons trouvée $\left(6,77 \mathrm{Wm}^{-2}{ }^{0} \mathrm{C}^{-1}\right)$, les valeurs de 1'isolation totale (air + tissus) données par Mount (I963b: $0, \mathrm{I}_{9}{ }^{\circ} \mathrm{C} \cdot \mathrm{m}^{2} \cdot \mathrm{W}^{-1}$ ) conduisent à une valeur de $\mathrm{C}_{i}$ de $23,6 \mathrm{Wm}^{-2}{ }^{0} \mathrm{C} 1$, comprise entre nos valeurs sur porcelets séchés et non séchés. Cette convergence est intéressante, car elle montre que nous avons estimé correctement la somme des résistances à la diffusion de la chaleur $\left(\frac{\mathrm{I}}{\mathrm{C}_{i}}+\frac{\mathrm{I}}{\mathrm{C}_{r}+\mathrm{C}_{c}}\right)$.

Rappelons en outre que $\mathrm{C}_{i}$ ne semble pas lié au poids des animaux, contrairement à la production de chaleur.

En conclusion, résumons les résultats principaux se dégageant de ce travail :

- l'hypothèse d'une régulation thermique principale au niveau de la peau (frisson) déjà formulée (Dussuel et BERBIGIER, I978), est confortée par l'allure des courbes (fig. I);

- les fluctuations des valeurs des températures rectales et cutanées autour de leur moyenne sont, à temps donné après la naissance, liées de façon significative à 1'humidité initiale de la peau, au poids de naissance et à la température rectale initiale. Des modèles statistiques minima permettent de prévoir les températures corporelles atteintes;

- cette prévision présente un intérêt dans la mesure où la température ambiante $\left(\mathrm{I} 7,5^{\circ} \mathrm{C}\right)$ est très proche du minimum supportable par un porcelet nouveau-né (NICHELMANN, BARNick, I,ys, I974).

Or les nouveau-nés, en général, ne têtent, ne se groupent et n'utilisent le chauffage d'appoint (lampes infra-rouge), qu'au bout d'une à plusieurs heures. Les conditions d'expérience présentes correspondent donc à la réalité zootechnique.

On peut alors comparer la vitesse de chute de la température rectale (entre $\mathrm{T}_{r} \mathbf{1 0}^{\mathbf{1 0}}$ et $\mathbf{T}_{r}{ }^{0}$ ) à la vitesse critique (MounT, I963a) au delà de laquelle le porcelet ne survit pas, et prévoir ainsi le devenir d'un animal.

- D'ores et déjà, on peut dire que la régulation thermique cutanée n'est effective que pour les animaux des deux classes de poids supérieures (I 200I $400 \mathrm{~g}$ et plus de I $400 \mathrm{~g}$ ); dans les autres cas, la température de la peau diminue avec le poids.

- Les estimations de la production de chaleur et des coefficients d'échanges montrent essentiellement l'effet bénéfique d'un séchage initial sur la vitalité du nouveau-né (thermogenèse et circulation sanguine).

D'une façon générale, on note la grande importance des facteurs physiques liés à l'animal (poids, conditions thermiques initiales, essuyage de la peau à la mise-bas). Nous démontrons par ailleurs (BERBIGIER et DUSSUEL, I977) que les effets constatés sont explicables par des modèles purement physiques : il semble donc que l'animal est réellement à la limite de ses possibilités physiologiques, et 
qu'il ne puisse réagir contre l'agression du milieu en modulant sa production de chaleur et sa vasoconstriction. La température des porcheries de maternité ( $\mathrm{I} 7^{\circ} \mathrm{C}$ ) est probablement trop basse pour les nouveau-nés.

$$
\text { Accepté pour publication en Fívricr } 1978 .
$$

\section{Summary}

\section{Measurement of thermal exchanges in newborn piglets by means of the energy balance method}

The influence of some characteristics of newborn piglets (weiglit, dry-wiping of the skin at birth, initial rectal and skin temperatures, sex) on the kinetics of rectal and skin surface temperatures during the first hour after birth, was studied at constant ambient temperature (I $7-18^{\circ} \mathrm{C}$ ) and relative humidity (50-6o per cent). Seventy-two piglets from $I$ I sows were involved in the experiment; about one half of the animals was wiped dry at birth and the other one was left wet. Temperature measurements were made at birth and at ro min. intervals up to $r$ hour of age.

Skin surface temperature first decreased by $7^{-9}{ }^{\circ} \mathrm{C}$ and then reached a plateau at 10 min. Rectal temperature decreased by $2-3^{\circ} \mathrm{C}$ and reached a plateau at about $30 \mathrm{~min}$ (fig. I). Skin wiping increased the two equilibrium temperatures by about ${ }^{\circ}{ }^{\circ} \mathrm{C}$. An increase in weight of $0.1 \mathrm{~kg}$ corresponded to an increase in rectal and surface temperatures of $0.3^{8}{ }^{\circ} \mathrm{C}$ and $0.3{ }^{\circ} \mathrm{C}$, respectively (fig. 1 ).

Table I shows that ambient climatic conditions were roughly the same for the different weight and treatment ("dry" or "wet" groups). Accordingly, ambient temperature and relative humidity were not taken into account in the statistical model used. This model shows that sex and sow effect had little or no significance (Table 2). Prevision of skin and rectal temperatures at $x$ and 60 min was obtained by reduced linear models. The influence of weight, skin-wiping and initial temperature was generally highly significant $(P<0.001)$. Though ambient climatic conditions were not taken into account, this model is of interest because an ambient temperature of $17-18{ }^{\circ} \mathrm{C}$ is close to the air temperature corresponding to summit metabolism, i.e. the maximum level of cold beyond which the piglet would suffer from hypometabolism. Sensible heat loss and tissue conductance were calculated on the basis of skin exchange coefficient estimates $\left(\mathrm{C}_{c}+\mathrm{C}_{r}=6,77 \mathrm{~W} \cdot \mathrm{m}^{-20} \mathrm{C}^{-1}\right)$.

Wiping the skin dry led to higher heat loss (i.e. heat production) and tissue conductance, which increased with age.

\section{Références bibliographiques}

BHRBigier P., i975. Sur la mesure des échanges de chaleur au niveau de la peau des porcs élevés sous abri par la méthode du bilan d'énergie. Ann. Zootech., 24, 4I 3-422.

BFRBIGIER P., 1978. Échanges thermiques des animaux d'élevage en fonction des paranlètres climatiques. Bull. Tech. d'Inf. (A paraître).

BrRbigier P. et DUSSUfi, A., I977. Étude des cinétiques de température et du confort thermique du porcelet pendant 1'heure suivant la mise-bas. In: "Energétique et thermique de l'homme dans son environnement $)$. Rencontre SFT. Strasbourg.

DE I A PORTE DES VAUX H. et AUMAITRE A., I967. Influence du milieu thermique sur l'évolution de la température rectale et de la glycémie chez le porcelet nouveau-né. Ann. Zootech., 16, 235245 .

DUSSUE, A. et BERRIGIER P., I978. Influence de la température ambiante sur les cinétiques des températures corporelles et le confort thermique des porcelets nouveau-nés. Ann. Zootech. 27, 83-93.

Joyce J. P., Braxter K. L. et PARK C., I966. The effect of natural out-door environment on the energy requirement of sheep. Res. Vet. Sci., 7, 342-359.

Monteith J. I., 1973. Principles of environmental physics. Edward Arnold ed., London, $24 \mathrm{I}$ p. 
Mount L. E., I962. Evaporative heat loss in the new-born pig. J. Physiol. Lond., 164, 274-281. Mount L. E., I963a. Responses to thermal environment in new-born pigs. liedn. Proc., 22, $818-823$.

Mount L. E., I963b. The thermal insulation of the new-born pig. J. Physiol. Lond., 168, 698705.

Mount L. E., 1967. The heat loss from new-born pigs to the floor. Res. Vet. Sci., 8, I75-1 86.

Movvr L. E., 1968. The climatic physiology of the pig. Edward Arnold ed., London, 27I p.

NeWLAND H. W., MC MILLISN W. $\mathrm{X}$, and ReINEKE E. P., 1952. Temperature adaptation in the baby pig. J. Anim. Sci., 11, I18-133.

NICHEL,MANN M., BARNiCK H. G., Lys L., I974. Untersuchungen zum Wärmehaushalt des neugeborenen Ferkels. I. Mitt : Wärmeproduktion. Mh. Vet.-Med., 26, r37-150.

Padalikova D., Holub A., Jezkova D., 1972. Glycogène dans le placenta et les tissus fœtaux des porcelets dans le dernier tiers de la vie intra-utérine (en Tchèque). Vet. Med., 45, II, 649656.

Stanton H. C., Brown L. J., MUeiLek R. L., 1973. Interrelationships between maternal and neonatal factors and thermoregulation in fasted neonatal swine (Sus domesticus). Comp. Biochem. Physiol., 44A, 97-105. 